Key words: enhancement of heat transfer, dream pipe, conjugate heat transfer, laminar oscillatory flow, hydromagnetic flow, liquid metals

\title{
STUDY OF CONJUGATE HEAT TRANSFER IN ELECTROMAGNETIC LIQUID METAL DREAM PIPE
}

\begin{abstract}
The combined effect of conjugation, external magnetic field and oscillation on the enhancement of heat transfer in the laminar flow of liquid metals between parallel plate channels is analyzed. In order to make our results useful to the design engineers, we have considered here only the wall materials that are widely employed in liquid metal heat exchangers. Indeed, all the results obtained through this mathematical investigation are in excellent agreement with the available experimental results. The effective thermal diffusivity $\kappa_{e}$ is increased by $3 \cdot 10^{6}$ times due to oscillation and that the heat flux as high as $1.5 \cdot 10^{10}\left(\mathrm{~W} / \mathrm{m}^{2}\right)$ can be achieved. Based on our investigation, we have recommended the best choice of liquid metal heat carrier, wall material and its optimum thickness along with the optimum value of the frequency to maximize the heat transfer rate. At the optimum frequency, by choosing a wall of high thermal conductivity and optimum thickness, an increase of $19.98 \%$ in $\kappa_{e}$ can be achieved. Our results are directly relevant to the design of a heat transfer device known as electromagnetic dream pipe which is a very recent development.
\end{abstract}

\section{Introduction}

The rapid changes in modern technology quite often demand the scientists and engineers to deal with very high temperature and hence there is an ever increasing need to develop devices for enhancement of heat transfer. Heat pipe is one such device. The interest in analytical studies and experimental designs of heat pipes started growing rapidly following the seminal publication by Grover et al. [1]. Kurzweg [2] designed a heat transfer device called dream pipe, for the enhancement of heat transfer, in which heat is transported from a hot to a cold reservoir by means of sinusoidal oscillations of viscous fluid contained within open ended capillary

\footnotetext{
${ }^{1}$ Department of Mathematics, Amrita School of Engineering, Coimbatore, Amrita Vishwa Vidyapeetham, Amrita University, India. Emails: p_puvaneswari@cb.amrita.edu, k_shailendhra@cb.amrita.edu
} 
tubes connecting the reservoirs. It should be noted here that the problem on the enhancement of heat transfer by sinusoidal oscillations of the fluid is called a dream pipe problem and Kurzweg [3] established that the same heat transfer process can take place in the oscillatory flow in an array of parallel plate channels. Heat transfer through capillary tubes connecting two fluid reservoirs having different temperatures was examined analytically and experimentally by Kurzweg and Ling de Zhao [4]. Effective thermal diffusivity is increased up to 17900 times than that in the non oscillation case. Ozawa and Kawamoto [5] who carried out a numerical simulation and thermal flow visualization of the dream pipe provided phenomenological explanations of the fundamental heat transfer mechanism in this novel heat transfer process. They concluded that the enhancement of heat transfer is mainly owing to the lateral diffusion of heat, accumulation capacity of heat in a region of depth of penetration formed near the wall and convective motion forced by the oscillation. Similar explanation was also rendered earlier by Kurzweg [6].

Indeed, this oscillation technique is based upon the periodic longitudinal convective and lateral diffusive thermal energy transport in the presence of a constant axial temperature gradient. Since this heat transfer process involves no net convective mass transfer, this is suitable for the removal of heat from hazardous substances like radioactive fluids [3]. Also, since devices based on this thermal pumping process are driven by external oscillations, it is possible to construct devices based on the technique of thermal valves which find applications in the field of cryogenics [6]. It also finds applications in the accelerated cooling devices for removing heat in combustion processes [3]. Some more remarkable advantages of dream pipes are explained elaborately in [2] and [7].

Incidentally, Kurzweg [3] suggested that it is better to use the liquid metals in the heat exchangers since their density, temperature gradient and specific heat capacity are very large. Heat transmission by oscillating the liquid metals is a topic of current interest owing to its tremendous applications in transporting thermal energy from Sterling and Brayton engines [8,9] and cooling of space nuclear reactors [10], high power electrical and electronic equipments. Further details on the designs of heat removal devices using liquid metals for fission surface power for Mars Moon and surface missions are well explained in [11].

Further, in these heat pipes with $\mathrm{Na}, \mathrm{NaK}$ and $\mathrm{K}$, the only relevant and mostly used wall materials are nickel, niobium and stainless steel $[12,13]$. In such heat transfer devices, not only the fluid properties but also the properties of the wall such as its thermal conductivity and thickness also influence the heat flux transported by the fluid and hence the problem becomes a conjugate heat transfer problem. Further, if the wall thickness is equal to or larger than the hydraulic diameter, the effect of heat conduction in the wall on the heat flux transported in the fluid cannot be neglected, and hence the level of conjugation becomes predominant [14]. Therefore, in the present investigation, as we are considering the channels with small hydraulic diameters, the effect of conjugation has to be considered. 
Several authors have considered the effect conjugation on the augmentation of heat transfer in the laminar and oscillatory flows. Kurzweg and de Zhao [4] investigated the effect of wall thermal conductivity on the effective thermal diffusivity of the fluid in a circular tube with infinite wall thickness and showed that the large amount of heat is transported when the frequency and amplitude are large. Kurzweg [3] analyzed the hydrodynamics of enhanced longitudinal heat transfer through a sinusoidally oscillating viscous fluid in an array of thermally conducting parallel plate channels analytically. Even though it is modelled as a conjugate heat transfer problem, the conjugation effect has not been carried out since the ratio of wall to fluid thickness is fixed as two and the wall and the fluid are considered to have similar thermal properties. Kaviany [15] extended the above problem of Kurzweg by including the effects of viscous dissipation, the presence of harmonics other than the fundamental harmonics, channel spacing and wall thickness and analysed the effect of wall thickness. Inaba et al. [16] examined the effect of conjugation on the heat transfer enhancement in an oscillatory flow through a circular pipe with conducting wall. Puvaneswari and Shailendhra [17] studied the effect of conjugation on the heat flux transported in a laminar liquid metal flow past a thermally conducting and sinusoidally oscillating infinite flat plate and concluded that the heat flux is increased by $46.14 \%$ by increasing the wall thickness to an optimum value.

The variations in the heat transfer characteristics with respect to the tube wall thickness were analysed by Kaviany [18] in an oscillatory flow through capillary tubes connecting two reservoirs having different temperatures. Inaba et al. [19] investigated the case of enhancement of heat transfer in the flow through bundles of conductive pipes of various regular polygonal cross sections including a circle. Takahashi [20] proposed a double-pipe heat exchange model to analyse the effect of oscillation on the effective thermal diffusivity of the fluid. However, in all these problems the authors have not considered the effect of conjugation.

In recent years, there is a great resurgence in the investigation of heat transfer in hydromagnetic oscillatory flows of liquid metals as these investigations are essential to design liquid metal magnetohydrodynamics heat exchange devices. The electromagnetic version of dream pipe is becoming a topic of current interest owing to its tremendous applications. Masao Furukawa et al. [21] examined the feasibility of an electromagnetic driven dream pipe by the analyses from both thermal and electrical points of view and showed that this novel heat transfer device may possibly replace the existing mechanically-driven dream pipes. Heat transfer studies with magnetohydrodynamic liquid metal flows are of great interest in the conceptual design of fusion power reactor [22] as liquid metals have incomparable heat transfer characteristics such as high thermal conductivity, high melting point and boiling point which eliminates the probability of local boiling [23]. The mentioned properties of liquid metals make them a good choice for the vacuum environment of space. Moreover, in the design of lithium blankets of thermo-nuclear reactors, the liquid metal has to be circulated in the presence of a strong magnetic field [24]. 
Shailendhra and Anjali Devi [25] analysed the effect of transverse magnetic field on the heat transfer enhancement in the flow past an oscillating flat plate immersed within a viscous fluid. The spatial variation of axial heat flux in various time intervals of the oscillation cycle in the case of hydromagnetic liquid metal flows in dream pipe with insulated walls was investigated by Shailendhra and Anjali Devi [26]. Moreover, studies on the heat transfer in the hydromagnetic flow of liquid metals are important in the development and design of metallurgical plants and electromagnetic stirring devices [27].

Many experiments on hydromagnetic thermo-hydraulics were mainly carried out from 1970's to 80's and Kirillov et al. [28] have summarized all these works. For further details on the current status and future scope of Liquid Metal Magneto Hydrodynamic Heat Transfer Systems, one may refer to the survey article by Morley et al. [29].

Recently, Puvaneswari and Shailendhra [30] have investigated the effects of magnetic field, wall thickness and wall thermal conductivity on the enhancement of heat transfer in a hydromagnetic laminar flow of liquid metals past an oscillating and thermally conducting infinite flat plate.

Motivated by the above investigations and applications, it is proposed to extend the earlier of work of Kurzweg [3] by considering the combined effects of conjugation and a transverse magnetic field when the working fluids are exclusively liquid metals like $\mathrm{Na}, \mathrm{K}$ and $\mathrm{NaK}$. The walls are considered to be of finite thickness and made up of compatible materials like $\mathrm{Ni}, \mathrm{Nb}$, AISI 316 , which are commonly used in liquid metal heat pipes $[12,13]$.

To the best of the knowledge of the authors, this is the very first attempt to investigate the combined effects of conjugation, oscillation and magnetic field on the enhancement of heat transfer in the flow between parallel plates. This investigation provides phenomenological explanations about the effect of conjugation and magnetic field on the enhancement of heat transfer. The new insights gained will be of immense use in the design, construction, control and improvement of a corresponding Liquid Metal Magneto Hydrodynamic Heat Transfer systems that can be designed using this oscillation technique. Such a heat transfer system can possibly be employed in nuclear reactors and also can be coupled with solar thermal systems.

\section{Mathematical formulation and solution}

Consider the laminar flow of a liquid metal, induced by a sinusoidal pressure gradient, within an array of channels, bounded by a long thermally-conducting infinite parallel plates, connecting a hot and a cold reservoir. The $x$ axis is chosen to be the axis of fluid motion and the $y$ axis is chosen normal to it. A constant axial temperature gradient $\gamma=\frac{\partial T}{\partial x}$ is maintained both within the fluid and the plates. The thickness of the fluid layers and solid walls are assumed to be $2 a$ and $2 b$ 


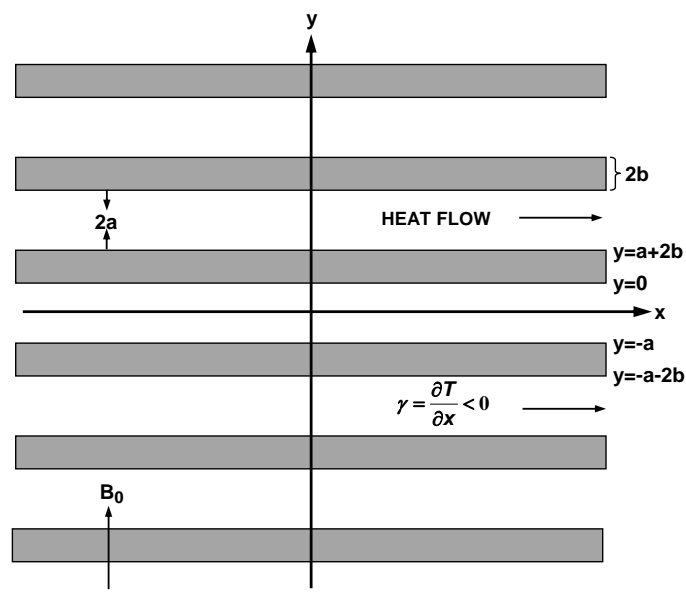

Fig. 1. Schematic representation of the problem

respectively. In parallel flows, neglecting the end effects is a fair approximation to reality provided the flow oscillation amplitude and the hydraulic diameter are both small compared to the plate length and hence the end effects present at points where the fluid enters and leaves the channels are neglected [31]. A uniform magnetic field with strength $B_{0}$ is applied in a direction transverse to the fluid motion.

The continuity equation is

$$
\nabla \cdot \vec{q}=0
$$

where $\vec{q}$ is the velocity of the fluid represented as $\vec{q}=[u, 0,0]$ which gives

$$
\frac{\partial u}{\partial x}=0
$$

and hence $u=u(y, t)$.

The momentum equation is

$$
\begin{gathered}
\rho_{f} \frac{\partial \vec{q}}{\partial t}=-\nabla p+\mu \nabla^{2} \vec{q}+\vec{j} \times \vec{B}, \\
\vec{B}=B_{0} \vec{j} \quad \text { and } \quad \vec{j} \times \vec{B}=-\sigma_{e} u B_{0}^{2 \vec{i}}
\end{gathered}
$$

where $\rho_{f}$ is the density of the fluid, $p$ - the pressure, $\mu$ - the dynamic viscosity, $B_{0}$ - the strength of transverse magnetic field, $\sigma_{e}$ - the electrical conductivity of the fluid, $\vec{j}$ - the current density vector, and $\vec{j} \times \vec{B}-$ the Lorentz force due to the transverse magnetic field.

The boundary condition for the velocity is

$$
u=0, \quad y=a .
$$

It must be noted here that the induced magnetic field is neglected in equation (3). This is because, under laboratory conditions, in the flow of liquid metals, 
induced magnetic field cannot be realized unless the flow becomes turbulent [32]. That is, magnetic Reynolds number, which is the ratio of the induced magnetic field to the applied magnetic field, in the case of liquid metal flows, is considerable enough to take into account the induced magnetic field only when the corresponding Reynolds number becomes very large that makes the flow to become turbulent. It should be noted here that the fluid flow is considered to be laminar in the present investigation.

The physical quantities are cast in non-dimensional form using $U=\frac{u}{U_{0}}, \eta=\frac{y}{a}$ where $U_{0}$ is the representative axial velocity and $\eta$ is the non-dimensional vertical coordinate.

Assuming $\frac{\partial p}{\partial x}=\left[A_{0} e^{i \omega t}\right]_{R}$ and accordingly $U(\eta, t)=\left[f(\eta) e^{i \omega t}\right]_{R}$ where $\omega$ is the frequency of oscillation and $A_{0}$ is the magnitude of the pressure gradient, from equations (3) and (4) we obtain

$$
f(\eta)=\frac{i \lambda}{\alpha^{2}-i M^{2}}\left[1-\frac{\cosh (C \eta)}{\cosh (C)}\right]
$$

where $\lambda=\frac{a^{2} A_{0}}{v \rho U_{0}}$ - the non-dimensional magnitude of imposed sinusoidal pressure gradient, $M=\sqrt{\frac{\sigma_{e} a^{2} B_{0}^{2}}{\rho v}}-$ Hartmann number, $\alpha=\sqrt{\frac{a^{2} \omega}{v}}-$ Womersley number and $C=\sqrt{M^{2}+i \alpha^{2}}$.

The tidal displacement $\Delta x$ is defined as

$$
\Delta x=U_{0}\left|\int_{\frac{-\pi}{2 \omega}}^{\frac{\pi}{2 \omega}} e^{i \omega t} d t \int_{0}^{1} f(\eta) d \eta\right|=\frac{2 U_{0} \lambda}{\omega}\left|\frac{1}{\alpha^{2}-i M^{2}}\left[1-\frac{\tanh C}{C}\right]\right|
$$

The heat equation in the fluid and solid are given by

$$
\begin{gathered}
\frac{\partial T_{f}}{\partial t}+\gamma\left[U_{0} f(\eta) e^{i \omega t}\right]_{R}=\frac{\kappa_{f}}{a^{2}} \frac{\partial^{2} T_{f}}{\partial \eta^{2}}, \quad 0<\eta<1 \\
\frac{\partial T_{s}}{\partial t}=\frac{\kappa_{s}}{a^{2}} \frac{\partial^{2} T_{s}}{\partial \eta^{2}}, \quad 1<\eta<1+2 \varepsilon, \quad \varepsilon=\frac{b}{a}
\end{gathered}
$$

where $T_{f}, \kappa_{f}$ and $T_{s}, \kappa_{s}$ are the temperature distribution and thermal diffusivity of the fluid and solid respectively.

As liquid metals have low Prandtl numbers, viscous dissipation term is neglected in equation (6). Following the lines of Kurzweg [6] it is to be noticed that 
the axial temperature gradient $\frac{\partial T}{\partial x}$ is small compared to the very large time dependent transverse temperature gradient $\frac{\partial T}{\partial y}$ that exists during most of the sinusoidal cycles which means that $\frac{\partial^{2} T}{\partial y^{2}}$ is much larger than $\frac{\partial^{2} T}{\partial x^{2}}$ and hence $\frac{\partial T}{\partial x}$ is taken as $\gamma$ which is actually the time averaged value of $\frac{\partial T}{\partial x}$.

As the heat flow is symmetrical with respect to $x$ axis, there is no heat flow along y direction across planes located in the middle of the fluid channels and in the middle of the bounding walls so that

$$
\begin{gathered}
\left(\frac{\partial T_{f}}{\partial \eta}\right)_{\eta=0}=0, \\
\left(\frac{\partial T_{s}}{\partial \eta}\right)_{\eta=1+\varepsilon}=0 .
\end{gathered}
$$

Both the temperature and the heat flux are continuous on the fluid-solid interface at $\eta=1$ so that

$$
\begin{aligned}
T_{f}(1) & =T_{s}(1) \\
k\left(\frac{\partial T_{f}}{\partial \eta}\right)_{\eta=1} & =\left(\frac{\partial T_{s}}{\partial \eta}\right)_{\eta=1}, \quad k=\frac{k_{f}}{k_{s}},
\end{aligned}
$$

where $k_{f}$ and $k_{s}$ represent the thermal conductivities of the fluid and the wall respectively.

In fact, it has been firmly established that the effect or level of conjugation, in general, is more pronounced in the case of unsteady, laminar flow of low Prandtl number fluids such as liquid metals [33] and the present problem satisfies all these requirements for a typical conjugate heat transfer problem.

To solve (6) and (7) for $T$, a temperature distribution of the following form [34] is assumed for both the fluid and the solid:

$$
T[x, \eta, t]=\gamma\left[x+a g(\eta) e^{i \omega t}\right]_{R}
$$

It should be noted that this form of solution is locally valid that has a physically realistic time averaged constant axial temperature gradient and that it exhibits a time dependent cross-stream variation in temperature [3]. This form has been already used by many authors like [4, 6, 7, 15-17] and [30].

From equations (6), (7) and (12) it is evident that

$$
g_{f}^{\prime \prime}(\eta)-i \alpha^{2} \operatorname{Pr} g_{f}(\eta)=\operatorname{Pef}(\eta), \quad 0<\eta<1,
$$




$$
g_{s}^{\prime \prime}(\eta)-i \alpha^{2} \operatorname{Pr} \sigma g_{s}(\eta)=0, \quad 1<\eta<1+2 \varepsilon
$$

where $P e=\frac{a U_{0}}{\kappa_{f}}$, Peclet number.

Solving (13) and (14) subject to conditions (8)-(11) it is found that

$$
\begin{aligned}
& g_{f}(\eta)=\operatorname{Pe} \lambda\left[a_{3} b_{1}(\eta)+a_{4}+a_{5} b_{2}(\eta)\right] \\
& g_{s}(\eta)=\operatorname{Pe} \lambda\left[a_{1}\left(a_{2}-C\right)\right] b_{3}(\eta)
\end{aligned}
$$

where the constants defined in the above expression are given in the appendix.

\subsection{Effective (enhanced) thermal diffusivity}

The effective (enhanced) averaged thermal diffusivity $\kappa_{e}$, can be defined by the equality [3]

$$
-\kappa_{e} \gamma=\frac{\omega}{2 \pi} \int_{0}^{\frac{2 \pi}{\omega}} d t \int_{0}^{1}[T[x, \eta, t]]_{R}\left[U_{0} f(\eta) e^{i \omega t}\right]_{R} d \eta
$$

where the subscript $R$ represents the real part of the terms shown. It is to be noted here that the quantities in this integrand do not average out to zero when integrated over one period of oscillation, so that there will be a net heat flow, although, obviously the time average of the velocity will be zero, so that there can be no net accompanying mass transport. However, there will be some axial diffusion mass transfer but this will be small for the fluids with high Schmidt number such as liquid metals [3].

Using (5) and (15) and performing the time integration in (17), the ratio of enhanced thermal diffusivity to the molecular thermal diffusivity is obtained as

$$
\frac{\kappa_{e}}{\kappa_{f}}=\frac{-1}{4} P e \int_{0}^{1}\left(f \overline{g_{f}}+g_{f} \bar{f}\right) d \eta
$$

where the bars represent the complex conjugate of the functions shown.

The effective ( enhanced ) thermal diffusivity $\kappa_{e}$ is obtained from (18) as

$$
\begin{gathered}
\kappa_{e}=\frac{-U_{0} a}{2} \int_{0}^{1}\left[\bar{f} g_{f}\right]_{R} d \eta \\
\text { where } \quad \bar{f} g_{f}=P e \lambda^{2} z_{1},
\end{gathered}
$$

where $z_{1}$ defined in the above expression is given in the appendix. 
Using (20) and the definition of tidal displacement $(\Delta x)$, the final expression for the effective thermal diffusivity is obtained as

$$
\kappa_{e}=\frac{-\omega(\Delta x)^{2} \alpha^{2} \operatorname{Pr}\left(M^{4}+\alpha^{4}\right)}{8 \times a_{14}} \int_{0}^{1}\left[\bar{f} g_{f}\right]_{R} d \eta
$$

where the expression for $a_{14}$ is given in the appendix.

The non-dimensional effective (enhanced) thermal diffusivity is given by

$$
\kappa_{e}^{*}=\frac{\kappa_{e}}{\omega(\triangle x)^{2}}
$$

\subsection{Transverse temperature difference}

The non-dimensional transverse temperature difference (NTTD), obtained from (12) is given by

$$
N T T D=\frac{T(x, 1, t)-T(x, 0, t)}{\gamma \triangle x}=\frac{\alpha^{2} \operatorname{Pr} g(\tau)}{2 h_{31}}, g(\tau)=h_{38} \cos \tau-h_{39} \sin \tau
$$

where $\tau=\omega t$. The constants occurring in the above equation are provided in the appendix.

\section{Results and discussion}

The objective of the present study is to analyse the combined effects of wall thickness, wall thermal conductivity, the Hartmann number and the Womersley number on the enhancement of heat transfer.

As stated above, $\mathrm{K}, \mathrm{Na}$ and $\mathrm{NaK}(22 \% \mathrm{Na}, 78 \% \mathrm{~K})$ are used as the heat exchange fluids and the wall is considered to be made up of $\mathrm{Ni}, \mathrm{Nb}$ and stainless steel of grade AISI 316. The operating temperature range $\left({ }^{\circ} \mathrm{C}\right)$ of heat pipes with $\mathrm{K}$, Na and $\mathrm{NaK}$ as the working fluids are 500-1000, 600-1200 and 425-825, respectively [13]. Hence, all the properties of the fluids and the wall materials are selected at $600^{\circ} \mathrm{C}$ for the sake of comparing the results. Accordingly, the following values are fixed for the various physical quantities. The thermal conductivities $k_{f}(\mathrm{~W} /(\mathrm{m} \mathrm{K}))$ and the thermal diffusivities $\kappa_{f}\left(\mathrm{~m}^{2} / \mathrm{s}\right)$ of $\mathrm{K}, \mathrm{Na}, \mathrm{NaK}(22 \% \mathrm{Na}, 78 \% \mathrm{~K})$ are 35.50 , $63.63,28.28$ and $6.765 \cdot 10^{-5}, 6.220 \cdot 10^{-5}, 4.408 \cdot 10^{-5}$ respectively. The Prandtl numbers of these fluids are $0.003143,0.004202$ and 0.00579 respectively. The electrical conductivities $(\mathrm{S} / \mathrm{m})$ of the same fluids are 1893939.39, 3215434.08 and 1468428.78. The thermal conductivities $k_{s}(\mathrm{~W} /(\mathrm{m} \mathrm{K}))$ of the above-mentioned wall materials $(\mathrm{Ni}, \mathrm{Nb}$ and stainless steel of grade AISI 316) are 65.6, 58.2, 18.3 and the corresponding thermal diffusivities $\kappa_{S}\left(\mathrm{~m}^{2} / \mathrm{s}\right)$ are $1.39071 \cdot 10^{-5}, 2.39969 \cdot 10^{-5}$ and $4.03893 \cdot 10^{-6}$, respectively [35]. In this context, $\varepsilon$ is varied from 0.4 to $2 ; \alpha$ is 
varied from $10^{-2}$ to 50 . The strength of magnetic field is varied from $0 \mathrm{mT}$ to 100 $\mathrm{mT}$, which is the usual range of magnetic field applied on the laminar flow of liquid metals [36]. Accordingly, the Hartmann number varies from 0 to 79, 0 to 86 and 0 to 62 for K, Na and NaK respectively.

Computing the value of $\kappa_{e}^{*}$ by varying the parameters as mentioned above, the optimum $\varepsilon$, the optimum $\alpha$ corresponding to the maximum value of $\kappa_{e}^{*}$ are obtained and presented in Table. 1 for all the fluids and solids under consideration, when $M=0$ and $M=60$. Maximum enhancement has been observed for the fluid $\mathrm{NaK}$ with the wall material $\mathrm{Ni}$ and the corresponding maximum value of the non-dimensional effective (enhanced) thermal diffusivity is 0.047 .

Optimum $\varepsilon$, optimum $\alpha$, the corresponding maximum value of $\kappa_{e}^{*}$

Table 1.

\begin{tabular}{|c|c|c|c|c|}
\hline \multicolumn{2}{|c|}{ Fluid } & \multicolumn{3}{|c|}{ Wall material } \\
\hline & & $\mathrm{Ni}$ & $\mathrm{Nb}$ & AISI 316 \\
\hline \multirow[t]{6}{*}{ K } & \multirow[t]{3}{*}{$M=0$} & 0.75 & 1 & 0.5 \\
\hline & & 25.73 & 24.7 & 22.69 \\
\hline & & 0.046 & 0.043 & 0.041 \\
\hline & \multirow[t]{3}{*}{$M=60$} & 0.75 & 1 & 0.5 \\
\hline & & 25.89 & 24.9 & 22.87 \\
\hline & & 0.045 & 0.042 & 0.040 \\
\hline \multirow[t]{6}{*}{$\mathrm{Na}$} & \multirow[t]{3}{*}{$M=0$} & 1 & 1.5 & 0.75 \\
\hline & & 19.6 & 17.25 & 14.91 \\
\hline & & 0.042 & 0.038 & 0.036 \\
\hline & \multirow[t]{3}{*}{$M=60$} & 1 & 1.5 & 0.75 \\
\hline & & 19.76 & 17.47 & 15.06 \\
\hline & & 0.041 & 0.037 & 0.034 \\
\hline \multirow[t]{6}{*}{$\mathrm{NaK}$} & \multirow[t]{3}{*}{$M=0$} & 1 & 1.5 & 0.5 \\
\hline & & 18.54 & 17.4 & 17.62 \\
\hline & & 0.047 & 0.044 & 0.042 \\
\hline & \multirow[t]{3}{*}{$M=60$} & 1 & 1.5 & 0.5 \\
\hline & & 18.90 & 17.64 & 17.9 \\
\hline & & 0.046 & 0.043 & 0.040 \\
\hline
\end{tabular}

Even though the analysis has been carried out and the discussions are made for a wide range of $M$, from 0 to 62 , the graphs are presented only for $M=10$ to minimize the number of graphs.

\subsection{Effect of magnetic field}

With the fluid $N a K$ and the wall material $N i$, it is found that at any frequency $\kappa_{e}^{*}$ decreases with increasing $M$ from 0 to 62 (Fig. 2a), which corresponds to the typical $M H D$ deterioration of heat transfer. One of the reasons for the reduction 

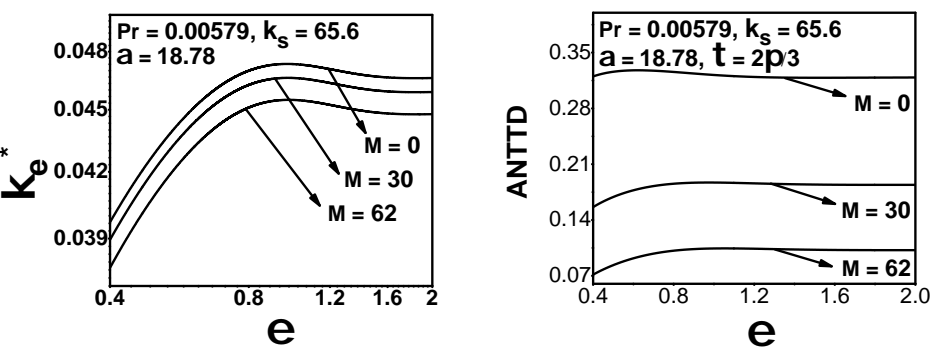

Fig. 2. Effect of $M$ on $\kappa_{e}^{*}$ and $A N T T D$ at optimum frequency

in $\kappa_{e}^{*}$ is the reduction in velocity due to the action of the Lorentz force. It is in good agreement with Shailendhra and Anjali Devi [25, 26] and Puvaneswari and Shailendhra [17]. Even though this analysis has been carried out for all possible values of $\alpha$, the results are presented only for optimum $\alpha$ to reduce the number of graphs. For the fluid $\mathrm{NaK}$ with the wall material $\mathrm{Ni}$ the optimum value of $\alpha$ is 18.78 , which is the average of the optimum values of $\alpha$ for $M=0,30,62$, by selecting $\varepsilon=1$, the optimum wall thickness which is found to be the same for each $M$ when the fluid is $\mathrm{NaK}$ and the wall material is $\mathrm{Ni}$.

Earlier, Kurzweg [6] established that the large transverse temperature gradient generated by the fluid vibration is the main reason for the heat transfer augmentation. To justify this result, the non-dimensional transverse temperature difference (NTTD) between $\eta=0$ and $\eta=1$ is calculated for all possible combination of fluids and solids. It should be noted here that larger the value of NTTD, larger in the conduction heat flux in the transverse direction. The sign of NTTD merely decides the direction in which the heat flows. That is, NTTD is positive when the heat flows from the wall to the fluid core and it is negative when the heat flows from the center of the pipe to the wall. Hence, all our discussions are based on the absolute value of NTTD denoted by ANTTD.

It is found that ANTTD decreases with increasing $M$ from 0 to 62 almost all the times. This result is shown in Fig. $2 \mathrm{~b}$ when $\tau=\pi / 3 . \tau$ is varied continuously from 0 to $2 \pi$ and similar observations were made for almost all the values of $\tau$. As the effects of $M$ on $A N T T D$ and $\kappa_{e}^{*}$ are similar, we conclude that the effect of $M$ on $\kappa_{e}^{*}$ depends on the effect of $M$ on ANTTD. This result has been already reported by Shailendhra and Anjali Devi [25] in the non-conjugate case of the hydromagnetic flow between counter-oscillating plates and by Puvaneswari and Shailendhra [17] in the conjugate case of hydromagnetic flow past an oscillating and thermally conducting flat plate.

\subsection{Effect of Womersley number}

The Womersley number $(\alpha)$ is defined as the square root of the ratio of the viscous penetration time scale to the oscillation time scale. Fig. 3a shows the effect of $\alpha$ on $\kappa_{e}^{*}$ for the fluid $\mathrm{NaK}$ with the wall material $\mathrm{Ni}$ for $M=10$. It is observed 
that when $\alpha$ increases, $\kappa_{e}^{*}$ increases initially and then it starts decreasing from a particular value of $\alpha$ onwards whatever may be the wall thickness. This result is valid for all values of $M$ from 0 to 62 . This is because, for small frequencies $\kappa_{e}^{*}$ is directly proportional to $\alpha^{2}$, while for high frequency, it is inversely proportional to $\alpha$, as observed by Kurzweg [3].
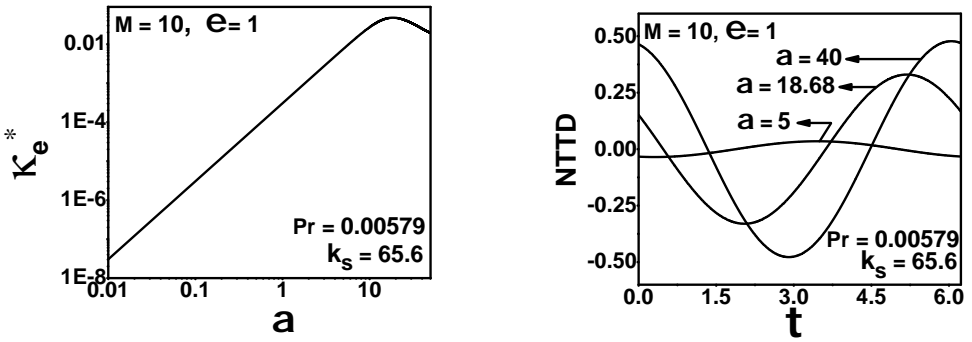

Fig. 3. Effect of $\alpha$ on $\kappa_{e}^{*}$ and NTTD at optimum $\varepsilon$

To understand the effect of $\alpha$ on $\kappa_{e}^{*}$, we examined the effect of $\alpha$ on the nondimensional transverse temperature difference (ANTTD) between the interface at $\eta=0$ and $\eta=1$. It is observed that, when $\alpha$ increases, ANTTD also increases at most all the times when $M=10$ and $\varepsilon=1$ (Fig. 3b). The same result is observed whatever may be the strength of magnetic field and wall thickness. As $\alpha$ increases both $\kappa_{e}^{*}$ and ANTTD increase up to an optimum value of $\alpha$ and hence the main reason for the enhancement of heat transfer up to optimum $\alpha$ is due to the increase in ANTTD. However, even though ANTTD increases after optimum $\alpha$, that is, in the case of large frequency, $\kappa_{e}^{*}$ decreases. This can be explained as follows.

Incidentally, $\beta^{2}=\frac{\alpha^{2} P r}{\kappa_{f}}$ is defined as the ratio of the thermal diffusion time scale to the oscillation time scale. When $\alpha \rightarrow \infty$ i.e., when $\beta \rightarrow \infty$, thermal diffusion time $>>$ oscillation period. Then most of the times the fluid oscillates between the reservoirs without carrying heat. In other words, the fluid behaves as a non-conductor. As a result of this, the axial convection heat flux decreases. Therefore, in the case of large frequency, even if ANTTD increases $\kappa_{e}^{*}$ decreases. Moreover, when $\alpha$ decreases to zero (i.e., when $\beta \rightarrow 0$ ) thermal diffusion time $<<$ oscillation period. In this case, the fluid oscillates between the reservoirs very slowly and the heat flows from the core to the wall very fast. As a result, there is a huge loss in transverse conduction heat flux which is reflected in $\kappa_{e}^{*}$. Therefore, when $\alpha$ decreases to zero $\kappa_{e}^{*}$ decreases. Further, physically at higher frequencies the velocity distribution in the channel changes and an inertial core with uniform velocity is formed around the axis of the channel. The presence of such an inertial core dominates over the viscous force and hence reduces the effectiveness of the fluid to store heat and, as a consequence, the heat flux transported by the fluid decreases [37]. 
Thus, in general, the effect of $\alpha$ on $\kappa_{e}^{*}$ depends on its effect on ANTTD only from $\alpha=0$ to $\alpha=\alpha$ optimum. Also, it is observed that the optimum value of $\alpha$ at which maximum $\kappa_{e}^{*}$ is obtained depends on the fluid chosen as well as the thermal conductivity and thickness of the wall.

\subsection{Effect of wall thickness}

The wall thickness plays a significant role in the enhancement of heat transfer because the thermal energy is stored in the wall and then released during one cycle of operation. Indeed, the walls absorb the heat energy from the hot fluid and give it off to the cold fluid, during various phases of the oscillation cycle. Following are the effects of $\varepsilon$ at low, optimum and high frequencies for $M=10$.

At low frequency as $\varepsilon$ increases $\kappa_{e}^{*}$ increases. On the other hand, at optimum frequency as $\varepsilon$ increases $\kappa_{e}^{*}$ increases initially but when $\varepsilon$ is increased further it has no effect on $\kappa_{e}^{*}$. However, when the frequency is very large $\kappa_{e}^{*}$ is independent of $\varepsilon$. These results are depicted in Fig. 4 for $M=10$. Similar observations were made for all the values of $M$ from 0 to 62 . These results have been already observed by [15-17] in the absence of magnetic field and by [30] in the presence of magnetic field. However, they have not rendered any physical justification for the above observation and hence we provide one here. The wall does not play any role on the heat transfer process in the fluid if the period of oscillation is less than the time required for heat to diffuse across the wall. That is, the wall thickness has no effect on $\kappa_{e}^{*}$ if $\frac{2 \pi}{\omega}<\frac{b^{2}}{\kappa_{s}}$ which is equivalent to $2 \pi<\alpha^{2} \varepsilon^{2} \operatorname{Pr} \sigma$. Therefore, given a fluid and a wall material, larger the value of $\alpha$ smaller will be the value of $\varepsilon$ for the above condition to be satisfied. This fact is illustrated in Fig. 4 for three different values of $\alpha$, one corresponding to the optimum frequency at which the maximum $\kappa_{e}^{*}$ is obtained, and the other two corresponding to the low and high frequencies. Moreover, at the optimum frequency and the optimum wall thickness, $\kappa_{e}^{*}$ is increased by $19.98 \%$ while using a wall with high thermal conductivity.

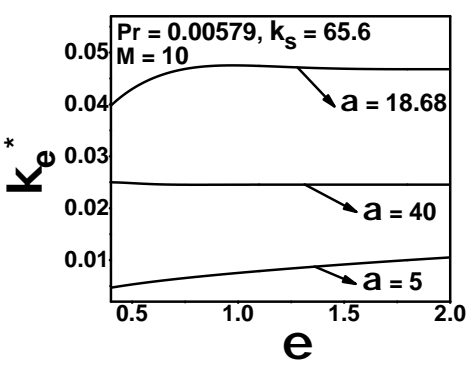

Fig. 4. Influence of $\varepsilon$ on $\kappa_{e}^{*}$ for three different $\alpha$ corresponding to the low, optimum and high frequencies 


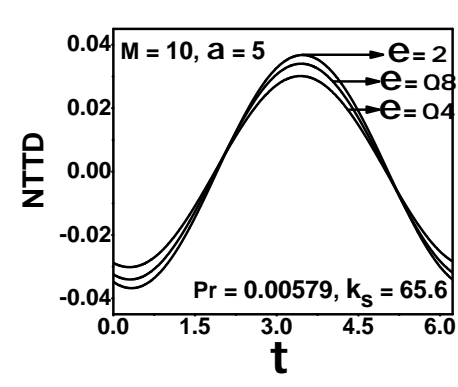

(a) At low frequency

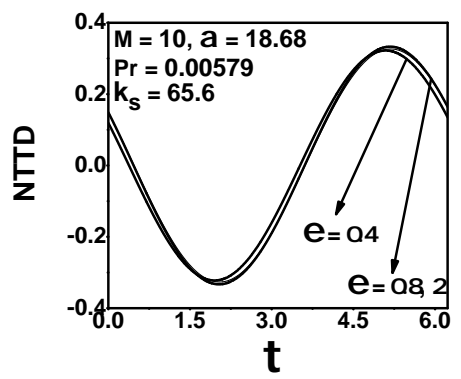

(b) At optimum frequency

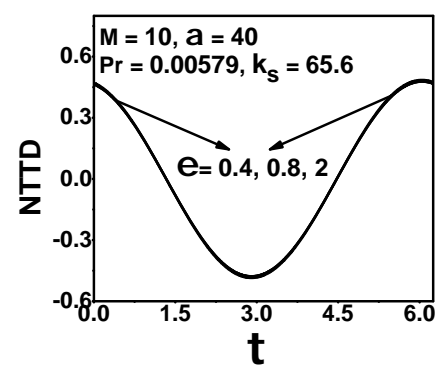

(c) At high frequency

Fig. 5. Effect of $\varepsilon$ on NTTD

It is also observed that, by and large, $A N T T D$ behaves in the same way as $\kappa_{e}^{*}$ due to the effect of $\varepsilon$ which is explained as follows. ANTTD is observed to increase as $\varepsilon$ increases when the frequency is small ( Fig. 5a). At optimum frequency as $\varepsilon$ increases the ANTTD also increases up to a certain $\varepsilon$ and the effect is saturated beyond that $\varepsilon$ (at some values of $\tau$ ) ( Fig. 5b). In the case of large frequency ANTTD becomes a constant with increasing $\varepsilon$ ( Fig. 5c). Thus, the effect of $\varepsilon$ on $\kappa_{e}^{*}$ almost depends on its effect on ANTTD when $M=10$. The same results are obtained for all values of $M$ from 0 to 62 . Also this result is consistent with the results reported by Puvaneswari and Shailendhra [30].

We further observe from Fig. 4 that $\kappa_{e}^{*}$ is independent of $\varepsilon$ when $\alpha$ is sufficiently large. This was shown analytically by [3]. We provide a physical justification for this result as follows. The wall thermal penetration distance is defined as $d=\sqrt{\frac{2 \kappa_{s}}{\omega}}$. For a given wall material, in the limiting case of $\alpha$ tending to infinity, this penetration distance tends to zero and hence there won't be any thermal interaction between the wall and the fluid and the wall thickness has no effect on $\kappa_{e}^{*}$. This can also be reasoned out as follows. In the case of large frequency, it is observed that ANTTD is independent of $\varepsilon$ as in Fig. $5 \mathrm{c}$. As the transverse temperature gradient plays a vital role in this heat exchange process and since ANTTD is independent of $\varepsilon, \kappa_{e}^{*}$ is also independent of $\varepsilon$ when the frequency is large. 


\subsection{Effect of the wall thermal conductivity}

Kaviany [18] mentioned that, in the case of oscillatory flows heat transfer rate is increased by the unsteady heat transfer between the pipe wall and the oscillating fluid. During the forward part of the oscillation, hotter fluid within the core causes a heat flow to the colder portions of the fluid within the boundary layers and to the colder solid walls bounding the fluid. During the other half of the cycle, heat from the hotter fluid in the boundary layers and the walls will diffuse into the fluid core, which is now colder [3]. Hence, the wall thermal conductivity plays a significant role in this heat transfer process.

Kurzweg and de Zhao [4] conducted experiments to investigate the effect of wall thermal conductivity on the effective thermal diffusivity of the fluid in a circular tube with infinite wall thickness by considering three interesting cases of zero, infinite wall conductivity and the case where the thermal conductivities and thermal diffusivities of the fluid and wall are equal. They reported that the performance of dream pipe is better when the thermal conductivity of the wall is higher. Inaba et al. [16] observed that the amount of heat transported by the fluid is increased when the conductivity of the pipe wall is increased. Following are the results obtained in the present study and the same are presented in Fig. 6 for $M=10$.

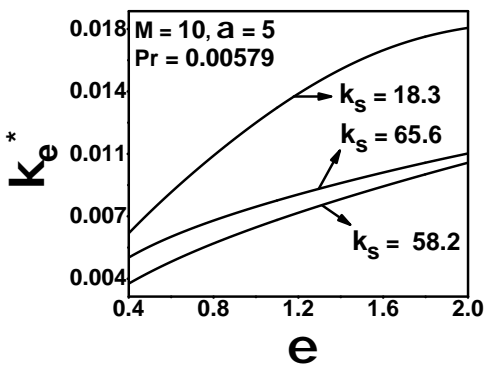

(a) At low frequency

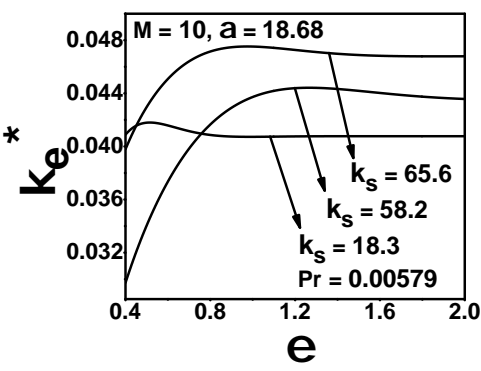

(b) At optimum frequency

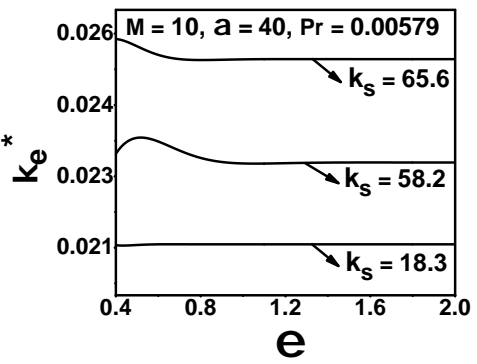

(c) At high frequency

Fig. 6. Effect of wall thermal conductivity on $\kappa_{e}^{*}$ 
In the case of large frequency, $\kappa_{e}^{*}$ increases as $k_{s}$ is increased whatever may be the value of $\varepsilon$ (Fig. 6c). Also, at optimum frequency, $\kappa_{e}^{*}$ increases as $k_{s}$ is increased (Fig. 6b). But this result is valid only beyond a particular value of $\varepsilon$. However, the above observations are not valid when the frequency is small (Fig 6a). Therefore, our results are in good agreement with the experimental results of Kurzweg and de Zhao [4] and the analytical results of Inaba et al. [16] beyond a particular wall thickness only when the frequency is either optimum or large.

The same trend is observed for ANTTD also. At low frequency, ANTTD doesn't increase in the increasing order of $k_{s}$ at any wall thickness (Fig. 7a). At optimum frequency, $A N T T D$ increases as $k_{s}$ increases only after a certain value of $\varepsilon$ (Figs. 8a, b). Moreover, at high frequency $A N T T D$ increases as $k_{s}$ is increased at any wall thickness (Fig. 7b).

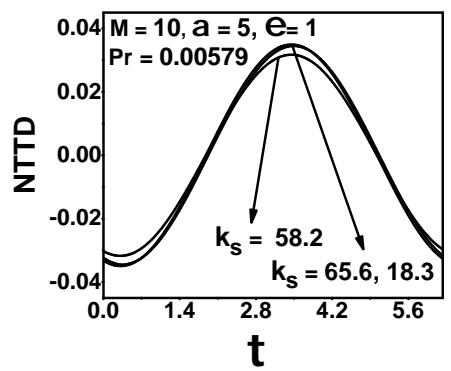

(a) At low frequency

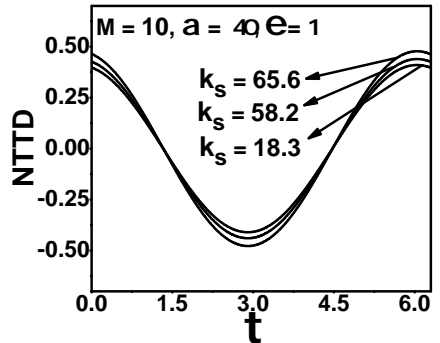

(b) At high frequency

Fig. 7. Effect of wall thermal conductivity on NTTD at low and high frequency

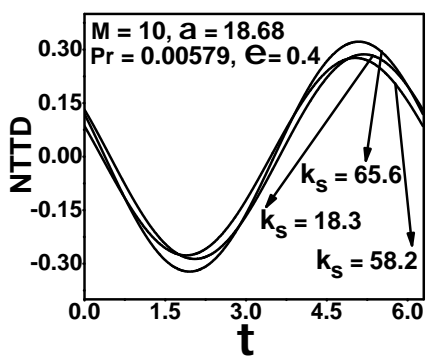

(a) $\varepsilon=0.4$

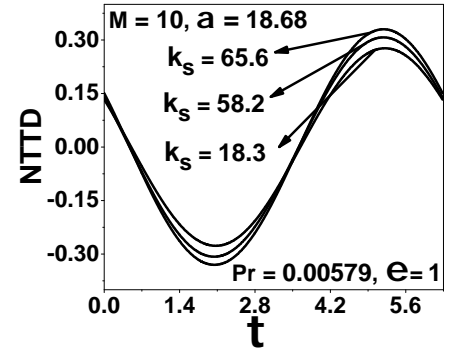

(b) $\varepsilon=1$

Fig. 8. Effect of wall thermal conductivity on NTTD at optimum frequency

Thus, from the above observations, it is concluded that the effect of $k_{s}$ on $\kappa_{e}^{*}$ depends on its effect on ANTTD and these effects depend on the range of values of $\alpha$ and $\varepsilon$ as explained above. The same observations are made for all values of $M$ from 0 to 62 . 
During simulation it is found that, for the other fluid and solid combinations all the results obtained in the present study are qualitatively the same but there is a quantitative change in the optimum values of $\alpha$ and $\varepsilon$.

\subsection{Total heat flux transported from the hot to the cold reservoir}

Total amount of heat transported between the axes of symmetry namely, $y=0$ $(\eta=0)$ ( in the fluid) and $y=a+b(\eta=1+\varepsilon)$ (in the solid) per unit time for a channel of width $W$ is the sum of the amount of heat transported by the fluid per unit time between $y=0$ and $y=a$ and the amount of heat transported by the solid per unit time between $y=a$ and $y=a+b$.

That is,

$$
Q(a+b) W=k_{e} a W \gamma+k_{s} b W \gamma
$$

where $k_{e}$ is the effective thermal conductivity of the fluid and $Q$ is the net heat transported $\left(\mathrm{W} / \mathrm{m}^{2}\right)$.

By neglecting the axial conduction in the solid since it is negligibly small as compared to the heat flux transported by the conduction in the fluid in the axial direction, the above equation gives the net heat flux transported as follows.

$$
Q=k_{e} \frac{a}{a+b} \gamma=\frac{\kappa_{e}^{*} \rho_{f} c_{f} \gamma}{1+\varepsilon}\left(\mathrm{W} / \mathrm{m}^{2}\right)
$$

where $\rho_{f}$ and $c_{f}$ represent the density and the specific heat capacity of the fluid, respectively.

The values of $Q$ corresponding to the maximum value of $\kappa_{e}^{*}$, and the optimum value of $\alpha$ and $\varepsilon$ (refer Table 1) are computed for various fluids and solids under consideration and are presented in Tables 2-3.

It is to be noted that, an axial heat flux of $1.3 \cdot 10^{10} \mathrm{~W} / \mathrm{m}^{2}$ was achieved experimentally by Kurzweg [2] with liquid Sodium heat carrier at an oscillation amplitude of $10 \mathrm{~cm}$ at a frequency of $30 \mathrm{~Hz}$. Moreover, Kurzweg [3] predicted an axial heat flux of $1.8 \cdot 10^{10} \mathrm{~W} / \mathrm{m}^{2}$ using pressurized water with $\rho_{f} c_{f}=1(\mathrm{cgs}$ units), temperature gradient $=\gamma=10^{\circ} \mathrm{C}, \mu=\sigma=1, \varepsilon=2, \omega=300 \mathrm{rad} / \mathrm{s}$ and $\Delta x=100 \mathrm{~cm}$. In order to calculate the maximum net heat flux transported $(Q)$ and to compare our results with those of Kurzweg [2, 3], we consider $\omega=300$ $\mathrm{rad} / \mathrm{s}$. In this case of high oscillation frequency $(\omega=300 \mathrm{rad} / \mathrm{s})$, there is a decrease in $Q$ when $M=60$ and the percentage of decrease varies from $2.1 \%$ to $4.01 \%$ (refer Table 2). As the percentage of reduction in $Q$ is very small, the magnetic field of strength up to $100 \mathrm{mT}$ can be effectively used as an additional control mechanism to maintain $Q$ at a desired level and to postpone the onset of transition to turbulence, thereby stabilizing the flow, if necessary. However, when $\omega$ is fixed as $300 \mathrm{rad} / \mathrm{s}$ the corresponding value of $a$, calculated from the optimum value of $\alpha$, becomes so small $(0.44 \mathrm{~mm} \leq a \leq 0.69 \mathrm{~mm})$, which is less than $3 \mathrm{~mm}$. This value of $a$ corresponds to mini-channel heat exchangers [38]. In this case, the pressure 
Maximum net heat flux $Q$ per unit area and the percentage of change in $Q$ due to the applied magnetic field when $\omega=300 \mathrm{rad} / \mathrm{s}$

\begin{tabular}{|c|c|c|c|c|}
\hline \multicolumn{2}{|c|}{ Fluid } & \multicolumn{3}{|c|}{ Wall material } \\
\hline & & $\mathrm{Ni}$ & $\mathrm{Nb}$ & AISI 316 \\
\hline \multirow[t]{2}{*}{$\mathrm{K}$} & $\begin{array}{l}M=0 \\
\left(10^{10} \mathrm{~W} / \mathrm{m}^{2}\right)\end{array}$ & 0.87 & 0.71 & 0.90 \\
\hline & $\begin{array}{l}M=60 \\
\left(10^{10} \mathrm{~W} / \mathrm{m}^{2}\right) \\
\%\end{array}$ & $\begin{array}{l}0.85 \\
-2.10\end{array}$ & $\begin{array}{l}0.70 \\
-2.19\end{array}$ & $\begin{array}{l}0.88 \\
-2.38\end{array}$ \\
\hline \multirow[t]{2}{*}{$\mathrm{Na}$} & $\begin{array}{l}M=0 \\
\left(10^{10} \mathrm{~W} / \mathrm{m}^{2}\right)\end{array}$ & 1.51 & 1.11 & 1.47 \\
\hline & $\begin{array}{l}M=60 \\
\left(10^{10} \mathrm{~W} / \mathrm{m}^{2}\right) \\
\%\end{array}$ & $\begin{array}{l}1.46 \\
-3.13\end{array}$ & $\begin{array}{l}1.07 \\
-3.32\end{array}$ & $\begin{array}{l}1.42 \\
-3.45\end{array}$ \\
\hline \multirow[t]{2}{*}{$\mathrm{NaK}$} & $\begin{array}{l}M=0 \\
\left(10^{10} \mathrm{~W} / \mathrm{m}^{2}\right)\end{array}$ & 1.09 & 0.82 & 1.29 \\
\hline & $\begin{array}{l}M=60 \\
\left(10^{10} \mathrm{~W} / \mathrm{m}^{2}\right) \\
\%\end{array}$ & $\begin{array}{l}1.05 \\
-3.84\end{array}$ & $\begin{array}{l}0.78 \\
-4.01\end{array}$ & $\begin{array}{l}1.24 \\
-3.93\end{array}$ \\
\hline
\end{tabular}

gradient required to drive the fluid through a channel of such a small hydraulic radius will become sufficiently large since $\partial p / \partial x$ is proportional to $1 / a^{4}$ for a constant mass flux. It is to be noted that, Kurzweg has recommended a hydraulic diameter of $3 \mathrm{~mm}$ with the frequency in the range of 2 to $50 \mathrm{~Hz}$, when the working fluid is liquid metal [2].

However, if we would like to have the dream pipe as a conventional heat pipe and take $a=3 \mathrm{~mm}$ [38], the angular frequency $\omega$ takes values only between 6.46 to $15.83(\mathrm{rad} / \mathrm{s})$. In this case, the percentage of decrease in $Q$ when $M=60$ varies from $0.1 \%$ to $1.57 \%$ (refer Table 3). Moreover, in this case, as the oscillation frequency varies from $1.03 \mathrm{~Hz}$ to $2.52 \mathrm{~Hz}$, a high rate of heat transfer is achieved even at low frequency. This result is in good agreement with Masao Furukawa et al. [21].

From Tables 2-3, it is inferred that the heat flux of order of $10^{10} \mathrm{~W} / \mathrm{m}^{2}$ can be achieved in dream pipe with liquid metals with compatible wall materials of finite thickness with $\omega=300 \mathrm{rad} / \mathrm{s}$ (as fixed by Kurzweg [3]) only if the dream pipe becomes a mini-channel heat exchanger and that the maximum heat flux obtained is $1.5 \cdot 10^{10} \mathrm{~W} / \mathrm{m}^{2}$. Moreover, in this case, $\kappa_{e}$ is increased by $31,39,383$ times than that existing in the absence of oscillations. However, as a conventional heat exchanger, the maximum heat flux that can be achieved in dream pipe is only of the order of $10^{8} \mathrm{~W} / \mathrm{m}^{2}$ and the maximum heat flux obtained is $5.62 \cdot 10^{8} \mathrm{~W} / \mathrm{m}^{2}$. In this case, $\kappa_{e}$ is increased by 1,03,600 times than that existing in the absence of oscillations. 
Table 3.

The optimum frequency of oscillation, the corresponding maximum net heat flux $Q$ per unit area and the percentage of change in $Q$ due to the applied magnetic field when $a=3 \mathrm{~mm}$

\begin{tabular}{|c|c|c|c|c|}
\hline \multicolumn{2}{|r|}{ Fluid } & \multicolumn{3}{|c|}{ Wall material } \\
\hline & & $\mathrm{Ni}$ & $\mathrm{Nb}$ & AISI 316 \\
\hline \multirow[t]{7}{*}{$\mathrm{K}$} & $M=0$ & & & \\
\hline & & 15.64 & 14.41 & 12.16 \\
\hline & $Q\left(10^{8} \mathrm{~W} / \mathrm{m}^{2}\right)$ & 4.54 & 3.43 & 3.66 \\
\hline & $M=60$ & & & \\
\hline & & 15.83 & 14.65 & 12.36 \\
\hline & $Q\left(10^{8} \mathrm{~W} / \mathrm{m}^{2}\right)$ & 4.50 & 3.41 & 3.63 \\
\hline & $\%$ & -0.91 & -0.56 & -0.78 \\
\hline \multirow[t]{7}{*}{$\mathrm{Na}$} & $M=0$ & & & \\
\hline & & 11.16 & 8.64 & 6.46 \\
\hline & $Q\left(10^{8} \mathrm{~W} / \mathrm{m}^{2}\right)$ & 5.62 & 3.18 & 3.17 \\
\hline & $M=60$ & & & \\
\hline & & 11.34 & 8.86 & 6.59 \\
\hline & $Q\left(10^{8} \mathrm{~W} / \mathrm{m}^{2}\right)$ & 5.54 & 3.16 & 3.13 \\
\hline & $\%$ & -1.57 & -0.86 & -1.50 \\
\hline \multirow[t]{7}{*}{$\mathrm{NaK}$} & $M=0$ & & & \\
\hline & & 9.75 & 8.59 & 8.8 \\
\hline & $Q\left(10^{8} \mathrm{~W} / \mathrm{m}^{2}\right)$ & 3.54 & 2.34 & 3.77 \\
\hline & $M=60$ & & & \\
\hline & $\omega$ & 10.13 & 8.83 & 9.09 \\
\hline & $Q\left(10^{8} \mathrm{~W} / \mathrm{m}^{2}\right)$ & 3.54 & 2.31 & 3.74 \\
\hline & $\%$ & -0.1 & -1.33 & -0.77 \\
\hline
\end{tabular}

\section{Comparison of our results with the results reported in the literature}

All the results obtained in the present investigation have been compared, in section 3, with the earlier works by [4, 15-18] and [30] in the non magnetic case and $[25,26]$ in the magnetic case, without the effect of conjugation.

Now, we would like to compare our results with those of Kurzweg [3], which is the basis for the present analysis. Kurzweg [3] analyzed the effect of Womersley number on the effective thermal diffusivity when the thermal conductivity and diffusivity of the fluid and wall are equal and $\varepsilon=2$. This result can be recovered from the present investigation by taking the limit as $M \rightarrow 0$ (nonmagnetic case) which is depicted in Fig. 9.

In the work of Kurzweg [3], the interface boundary condition should have been $g_{s}^{\prime}(\varepsilon+1)=0$ instead of $g_{s}^{\prime}(\varepsilon)=0$ (as given by Kurzweg [3]). Similarly, the formula for net heat flux per unit area should have been $Q=\frac{\kappa_{e} \rho_{f} C_{f} \gamma}{1+\varepsilon}$ and not 
$Q=\frac{\kappa_{e} \rho_{f} C_{f} \gamma}{\varepsilon}$ (as given by Kurzweg [3]). The curves drawn by us, incorporating the above conditions, given in Fig. 9 are neither qualitatively nor quantitatively different from those presented by Kurzweg [3] since $\kappa_{e}^{*}$ is independent of $\varepsilon$ for higher values of $\varepsilon$,viz; $(\varepsilon \geq 0.75)$.

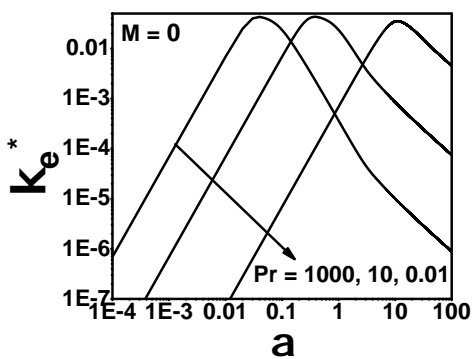

Fig. 9. Effect of $\alpha$ on $\kappa_{e}^{*}$ for three different Prandtl numbers when $\mu=\sigma=1$ and $\varepsilon=2$

\section{Conclusions}

Elaborate discussions along with appropriate physical explanations on the combined effects of conjugation and uniform transverse magnetic field on the enhancement of heat transfer in the hydromagnetic version of the dream pipe have been rendered in the previous section. Here we highlight only the results that are hitherto not reported in the literature that are useful to the research community.

- At any frequency, $Q$ decreases with increasing $M$, which corresponds to the typical MHD deterioration of heat transfer. In particular, when $M=60, Q$ decreases by about $2.10 \%$ to $4.01 \%$, respectively, depending on the fluid, the wall and the oscillation frequency.

- An axial heat flux of $1.5 \cdot 10^{10} \mathrm{~W} / \mathrm{m}^{2}$ can be achieved with $\omega=300 \mathrm{rad} / \mathrm{s}$ as stated by Kurzweg only when $0.44 \leq a \leq 0.69$, which corresponds to minichannel heat exchanger [38] and the effective thermal diffusivity is increased by $31,39,383$ times than that existing in the absence of oscillations. On the other hand, the maximum heat flux of $5.62 \cdot 10^{8} \mathrm{~W} / \mathrm{m}^{2}$ can be achieved in the case of dream pipe as a conventional heat pipe $(a=3 \mathrm{~mm})$ [38] and $\kappa_{e}$ is increased by 1,03,600 times than that existing in the absence of oscillations. Therefore, mini-channel heat exchangers with $\omega=300 \mathrm{rad} / \mathrm{s}$ can be used to achieve a heat flux of order of $10^{10} \mathrm{~W} / \mathrm{m}^{2}$.

- The optimum wall thickness, the corresponding optimum frequency of oscillation for achieving maximum value of $\kappa_{e}^{*}$ are presented.

- As $\varepsilon$ is increased up to a critical value which depends on the frequency of oscillation, the fluid and the wall material used, $\kappa_{e}^{*}$ is also increased. However, when $\varepsilon$ is increased beyond the critical value, $\kappa_{e}^{*}$ becomes constant. At the optimum frequency, by choosing a wall of high thermal conductivity, an 
increase of $19.98 \%$ in $\kappa_{e}^{*}$ can be achieved by optimizing the wall thickness.

When the frequency is very large, $\kappa_{e}^{*}$ is independent of wall thickness.

- As $k_{s}$ is increased $\kappa_{e}^{*}$ is also increased only after the optimum frequency.

- In general, the substantial increase in the transverse temperature gradient due to oscillation is the main cause for the enhancement of heat transfer. The effects of all the parameters on the effective thermal diffusivity depend on their effects on the absolute value of the transverse temperature gradient.

\section{A. Appendix}

$$
\begin{aligned}
& k_{1}=\sqrt{\frac{M^{2}+\sqrt{M^{4}+\alpha^{4}}}{2}} \quad k_{2}=\sqrt{\frac{\sqrt{M^{4}+\alpha^{4}}-M^{2}}{2}} \\
& a_{1}=\frac{-C}{\left(\alpha^{2} \operatorname{Pr}\right)\left(\alpha^{2}-i M^{2}\right)\left(i \alpha^{2} \operatorname{Pr}-C^{2}\right) \cosh \sqrt{i \operatorname{Pr} \alpha}} \\
& a_{2}=\frac{C \sqrt{\sigma} \tanh \sqrt{i \operatorname{Pr} \sigma} \alpha \varepsilon+\mu \alpha \sqrt{i \operatorname{Pr}} \tanh C}{\mu \tanh \sqrt{i \operatorname{Pr}} \alpha+\sqrt{\sigma} \tanh \sqrt{i \operatorname{Pr} \sigma} \alpha \varepsilon} \\
& a_{3}=a_{1} a_{2} \\
& a_{4}=\frac{-1}{\left(\alpha^{2} \operatorname{Pr}\right)\left(\alpha^{2}-i M^{2}\right)} \\
& a_{5}=\frac{-i}{\left(\alpha^{2}-i M^{2}\right)\left(C^{2}-i \alpha^{2} \operatorname{Pr}\right)} \quad a_{6}=\frac{\cosh \sqrt{i \operatorname{Pr}} \alpha}{\cosh \sqrt{i \operatorname{Pr} \sigma} \alpha \varepsilon} \\
& a_{7}=\cosh k_{1} \cos k_{2}, \quad a_{8}=\sinh k_{1} \sin k_{2} \\
& a_{9}=a_{7}-i a_{8} \quad a_{10}=a_{7}+i a_{8} \\
& a_{11}=\cosh 2 k_{1}+\cos 2 k_{2} \quad a_{12}=\sinh ^{2} 2 k_{1}+\sin ^{2} 2 k_{2} \\
& a_{13}=k_{1} \sinh 2 k_{1}+k_{2} \sin 2 k_{2} \quad a_{14}=1+\frac{\left[\left(a_{12} / a_{11}\right)-2 a_{13}\right]}{a_{11} \sqrt{M^{4}+\alpha^{4}}} \\
& b_{1}(\eta)=\cosh \sqrt{i \operatorname{Pr}} \alpha \eta \quad b_{2}(\eta)=\frac{\cosh C \eta}{\cosh C} \\
& b_{3}(\eta)=\cosh \sqrt{i \operatorname{Pr} \sigma} \alpha(\varepsilon+1-\eta) \quad b_{4}(\eta)=\cosh k_{1} \eta \cos k_{2} \eta \\
& b_{5}(\eta)=\sinh k_{1} \eta \sin k_{2} \eta \quad b_{6}(\eta)=b_{5}(\eta)+i b_{4}(\eta) \\
& b_{7}(\eta)=b_{4}(\eta)+i b_{5}(\eta) \\
& z_{1}=\left[a_{3} b_{1}(\eta)+a_{4}+\frac{a_{5}}{a_{10}} b_{7}(\eta)\right]\left[\frac{1}{\alpha^{2}+i M^{2}}\left(-i+\frac{b_{6}(\eta)}{a_{9}}\right)\right]
\end{aligned}
$$




$$
\begin{aligned}
& h_{1}=\alpha^{2} M^{2}(P r-2) \\
& h_{2}=M^{4}+\alpha^{4}(P r-1) \\
& h_{3}=\cosh \left(\sqrt{\frac{P r}{2}} \alpha\right) \cos \left(\sqrt{\frac{P r}{2}} \alpha\right) \\
& h_{4}=\sinh \left(\sqrt{\frac{P r}{2}} \alpha\right) \sin \left(\sqrt{\frac{P r}{2}} \alpha\right) \\
& h_{5}=h_{1} \times h_{3}-h_{2} h_{4} \\
& h_{6}=h_{1} \times h_{4}+h_{2} h_{3} \\
& h_{7}=\frac{k_{1} h_{5}+k_{2} h_{6}}{h_{5}^{2}+h_{6}^{2}} \\
& h_{9}=\frac{\sqrt{\sigma} \sinh (\sqrt{2 \operatorname{Pr} \sigma} \varepsilon \alpha)}{\cosh (\sqrt{2 \operatorname{Pr} \sigma} \varepsilon \alpha)+\cos (\sqrt{2 \operatorname{Pr} \sigma} \varepsilon \alpha)} \\
& h_{8}=\frac{-k_{1} h_{6}+k_{2} h_{5}}{h_{5} h_{5}+h_{6} h_{6}} \\
& h_{10}=\frac{\sqrt{\sigma} \sin (\sqrt{2 \operatorname{Pr} \sigma} \varepsilon \alpha)}{\cosh (\sqrt{2 \operatorname{Pr} \sigma} \varepsilon \alpha)+\cos (\sqrt{2 \operatorname{Pr} \sigma} \varepsilon \alpha)} \\
& h_{11}=\frac{\mu \sinh (\sqrt{2 \operatorname{Pr}} \alpha)}{\cosh (\sqrt{2 \operatorname{Pr}} \alpha)+\cos (\sqrt{2 \operatorname{Pr}} \alpha)} \\
& h_{12}=\frac{\mu \sin (\sqrt{2 \operatorname{Pr}} \alpha)}{\cosh (\sqrt{2 \operatorname{Pr}} \alpha)+\cos (\sqrt{2 \operatorname{Pr}} \alpha)} \\
& h_{13}=\frac{\mu \alpha \sqrt{\frac{P r}{2}}\left(\sinh \left(2 k_{1}\right)-\sin \left(2 k_{2}\right)\right)}{\cosh \left(2 k_{1}\right)+\cos \left(2 k_{2}\right)} \\
& h_{14}=\frac{\mu \alpha \sqrt{\frac{P r}{2}}\left(\sinh \left(2 k_{1}\right)+\sin \left(2 k_{2}\right)\right)}{\cosh \left(2 k_{1}\right)+\cos \left(2 k_{2}\right)} \\
& h_{15}=k_{1} h_{9}-k_{2} h_{10} \\
& h_{16}=k_{1} h_{10}+k_{2} h_{9} \\
& h_{17}=h_{13}+h_{15} \\
& h_{18}=h_{14}+h_{16} \\
& h_{19}=h_{9}+h_{11} \\
& h_{20}=h_{10}+h_{12} \\
& h_{21}=\frac{h_{17} h_{19}+h_{18} h_{20}}{h_{19}^{2}+h_{20}^{2}} \\
& h_{22}=\frac{-h_{17} h_{20}+h_{18} h_{19}}{h_{19}^{2}+h_{20}^{2}} \\
& h_{23}=h_{7} h_{21}-h_{8} h_{22} \\
& h_{24}=h_{7} h_{22}+h_{8} h_{21} \\
& h_{25}=-h_{1} a_{7}+h_{2} a_{8} \\
& h_{26}=-h_{1} a_{8}-h_{2} a_{7} \\
& h_{27}=\frac{k_{1} \sin h\left(2 k_{1}\right)+k_{2} \sin \left(2 k_{2}\right)}{a_{11}\left(k_{1}^{2}+k_{2}^{2}\right)} \\
& h_{28}=\frac{k_{1} \sin \left(2 k_{2}\right)-k_{2} \sin h\left(2 k_{1}\right)}{a_{11}\left(k_{1}^{2}+k_{2}^{2}\right)} \\
& h_{29}=\frac{\alpha^{2}\left(1-h_{27}\right)+M^{2} h_{28}}{M^{4}+\alpha^{4}} \\
& h_{30}=\frac{M^{2}\left(1-h_{27}\right)-\alpha^{2} h_{28}}{M^{4}+\alpha^{4}} \\
& h_{31}=\sqrt{h_{29}^{2}+h_{30}^{2}} \\
& h_{32}=h_{3}-1 \\
& h_{33}=h_{32} h_{23}-h_{4} h_{24} \\
& h_{34}=h_{4} h_{23}+h_{32} h_{24} \\
& h_{35}=a_{7}-1 \\
& h_{36}=h_{25} a_{8}-h_{35} h_{26} \\
& h_{37}=-h_{26} a_{8}-h_{35} h_{25} \\
& h_{39}=-\frac{h_{34}}{\alpha^{2} P r}+\frac{h_{37}}{h_{25}^{2}+h_{26}^{2}} \\
& h_{38}=\frac{-h_{33}}{\alpha^{2} \operatorname{Pr}}+\frac{h_{36}}{h_{25}^{2}+h_{26}^{2}}
\end{aligned}
$$




\section{Acknowledgements}

We gratefully acknowledge the comments of the reviewers which enabled us to improve the quality of the article.

Manuscript received by Editorial Board, November 07, 2016; final version, March 14, 2017.

\section{References}

[1] G.M. Grover, T.P. Cotter, and G.F. Erickson. Structures of very high thermal conductance. Journal of Applied Physics, 35(6):1990-1991, 1964. doi: 10.1063/1.1713792.

[2] H Kurzweg. Heat transfer device for the transport of large conduction flux without net mass transfer, May 27 1986. US Patent 4,590,993.

[3] U.H. Kurzweg. Enhanced heat conduction in oscillating viscous flows within parallel-plate channels. Journal of Fluid Mechanics, 156:291-300, 1985. doi: 10.1017/S0022112085002105.

[4] U.H. Kurzweg and L. de Zhao. Heat transfer by high-frequency oscillations: A new hydrodynamic technique for achieving large effective thermal conductivities. Physics of Fluids, 27(11):2624-2627, 1984. doi: 10.1063/1.864563.

[5] M. Ozawa and A. Kawamoto. Lumped-parameter modeling of heat transfer enhanced by sinusoidal motion of fluid. International journal of heat and mass transfer, 34(12):3083-3095, 1991. doi: 10.1016/0017-9310(91)90078-S.

[6] U.H. Kurzweg. Temporal and spatial distribution of heat flux in oscillating flow subjected to an axial temperature gradient. International journal of heat and mass transfer, 29(12):1969-1977, 1986. doi: 10.1016/0017-9310(86)90016-5.

[7] K. Shailendhra and S.P. AnjaliDevi. On the enhanced heat transfer in the oscillatory flow of liquid metals. Journal of Applied Fluid Mechanics, 4(2):57-62, 2011.

[8] P. Bouvier, P. Stouffs, and J.-P. Bardon. Experimental study of heat transfer in oscillating flow. International Journal of Heat and Mass Transfer, 48(12):2473-2482, 2005. doi: 10.1016/j.ijheatmasstransfer.2005.01.037.

[9] R.C. Tew and S.M. Geng. Overview of NASA supported Stirling thermodynamic loss research. Technical report, NASA Technical Reports Server (NTRS), 1992.

[10] M. El-Genk and J.-M. Tournier. Uses of liquid-metal and water heat pipes in space reactor power systems. Frontiers in Heat Pipes (FHP), 2(1):1-24, 2011. doi: 10.5098/fhp.v2.1.3002.

[11] R.W. Dyson, B. Penswick, M. Robbie, and S.M. Geng. Investigation of liquid metal heat exchanger designs for fission surface power. In Sixth International Energy Conversion Engineering Conference (IECEC)., pages 7-38, Cleveland, USA, 28-30 July 2009. doi: 10.2514/6.20085733 .

[12] L.R. Kelman, W.D. Wilkinson, and F.L. Yaggee. Resistance of materials to attack by liquid metals. Technical report, Argonne National Laboratory, 1950.

[13] D.A. Reay and P.A. Kew. Heat Pipes: Theory, Design and Applications. ButterworthHeinemann, 5 edition, 2006.

[14] K.D. Cole and Barbaros Çetin. The effect of axial conduction on heat transfer in a liquid microchannel flow. International Journal of Heat and Mass Transfer, 54(11):2542-2549, 2011. doi: 10.1016/j.ijheatmasstransfer.2011.02.007.

[15] M. Kaviany. Some aspects of enhanced heat diffusion in fluids by oscillation. International Journal of Heat and Mass Transfer, 29(12):2002-2006, 1986. doi: 10.1016/0017-9310(86)90022-0. 
[16] T. Inaba, G. Morita, and K.-I. Saitoh. Longitudinal heat transfer enhanced by fluid oscillation in a circular pipe with conductive wall. Heat Transfer - Asian Research, 33(2):129-139, 2004. doi: $10.1002 / \mathrm{htj} .10126$.

[17] P. Puvaneswari and K. Shailendhra. Enhancement of heat transfer in a liquid metal flow past a thermally conducting and oscillating infinite flat plate. Journal of Applied Fluid Mechanics, 9(3):1395-1407, 2016.

[18] M. Kaviany. Performance of a heat exchanger based on enhanced heat diffusion in fluids by oscillation: analysis. Journal of Heat Transfer, 112(1):49-55, 1990. doi: 10.1115/1.2910363.

[19] T. Inaba, M. Tahara, and K.-I. Saitoh. Longitudinal heat transfer in oscillatory flows in pipe bundles of various cross sections. JSME International Journal Series B Fluids and Thermal Engineering, 43(3):460-467, 2000. doi: 10.1299/jsmeb.43.460.

[20] Takahashi I. Axial heat transfer characteristics enhanced by oscillating fluid in thin tube (a newly proposed model and expression for effective thermal diffusivity). Transactions of the Japan Society of Mechanical Engineers Series B, 61(581):275-282, 1995. (in Japanese), doi: 10.1299/kikaib.61.275.

[21] M. Furukawa, M. Morishita, and S. Yokoyama. Feasibility study of electromagnetic driven dream pipe. International Journal of Heat and Mass Transfer, 83:212-221, 2015. doi: 10.1016/j.jheatmasstransfer.2014.11.072.

[22] A.K. Kalkan and G. Talmage. Heat transfer in liquid metals with electric currents and magnetic fields: the conduction case. International Journal of Heat and Mass Transfer, 37(3):511-521, 1994. doi: 10.1016/0017-9310(94)90086-8.

[23] J.S. Rao and H. Sankar. Magneto hydro-dynamics and heat transfer in liquid metal flows. In Marco Aurelio Dos Santos Bernardes, editor, Developments in Heat Transfer, chapter 4, pages 55-80. INTECH Open Access Publisher, 2011.

[24] H. Branover and C. Henoch. Control of friction pressure losses and heat transfer in turbulent liquid metal flows in magnetic fields. Magnetohydrodynamics, 29(4):329-340, 1994.

[25] K. Shailendhra and S.P. Anjali Devi. Heat transport along an oscillating flat plate in the presence of a transverse magnetic field. International Journal of Heat and Mass Transfer, 40(2):498-501, 1997. doi: 10.1016/0017-9310(95)00281-2.

[26] K. Shailendhra and S.P. Anjali Devi. Temporal and spatial distribution of heat flux in oscillating MHD flow subjected to an axial temperature gradient. In Proceedings of the International Conference on Recent Advances in Mathematics, pages 182-193, Gulbarga University, Gulbarga, India, 2005.

[27] J.F. Smith, M.-Y. Hsiao, T.F. Lin, and M.G. Willis. Magnetohydrodynamically enhanced heat transfer in a liquid metal system. Nuclear Engineering and Design, 125(2):147-159, 1991. 10.1016/0029-5493(91)90074-R.

[28] I.R. Kirillov, C.B. Reed, L. Barleon, and K Miyazaki. Present understanding of MHD and heat transfer phenomena for liquid metal blankets. Fusion Engineering and Design, 27:553-569, 1995. doi: 10.1016/0920-3796(95)90171-X.

[29] N.B. Morley, S. Smolentsev, L. Barleon, I.R. Kirillov, and M. Takahashi. Liquid magnetohydrodynamics - recent progress and future directions for fusion. Fusion Engineering and Design, 51:701-713, 2000. 10.1016/S0920-3796(00)00197-6.

[30] P. Puvaneswari and K. Shailendhra. Enhancement of heat transfer in a laminar hydromagnetic flow of a liquid metal past a thermally conducting and oscillating infinite flat plate. Heat Transfer-Asian Research, 2016. doi: 10.1002/htj.21233.

[31] D. Gedeon. Mean-parameter modeling of oscillating flow. Journal of Heat Transfer, 108(3):513-518, 1986. doi: 10.1115/1.3246964.

[32] Roberts P.H. An introduction to magnetohydrodynamics. American Elsevier Pub. Co., 1967.

[33] A.S. Dorfman. Conjugate problems in convective heat transfer. CRC Press, Boca Raton, USA, 2009. 
[34] P.C. Chatwin. On the longitudinal dispersion of passive contaminant in oscillatory flows in tubes. Journal of Fluid Mechanics, 71(3):513-527, 1975. doi: 10.1017/S0022112075002716.

[35] Y.A. Çengel and A.J. Ghajar. Heat and Mass Transfer. Fundamentals and Applications. McGraw-Hill, 5th edition, 2015.

[36] Z. Recebli, S. Selimli, and E. Gedik. Three dimensional numerical analysis of magnetic field effect on convective heat transfer during the mhd steady state laminar flow of liquid lithium in a cylindrical pipe. Computers \& Fluids, 88:410-417, 2013. doi: 10.1016/j.compfluid.2013.09.009.

[37] Kaviany M. Principles of convective heat transfer. Springer-Verlag, New York, 2nd edition, 2001.

[38] M. Ohadi, K. Choo, S. Dessiatoun, and E. Cetegen. Next Generation Microchannel Heat Exchangers. Springer, 2013. 